\title{
Research and Development of Ion Surfing RF Carpets for the Cyclotron Gas Stopper at the NSCL
}

\author{
A.E. Gehring ${ }^{1}$, M. Brodeur ${ }^{2}$, G. Bollen ${ }^{3}$, D.J. Morrissey ${ }^{3}$, S. Schwarz ${ }^{3}$ \\ ${ }^{1}$ Los Alamos National Laboratory, P.O. Box 1663, Los Alamos, NM 87544, USA, ${ }^{2}$ University of Notre Dame, Notre Dame, IN USA, ${ }^{3}$ National Superconducting \\ Cyclotron Laboratory, Michigan State University, 640 S. Shaw Lane, East Lansing, MI 48824, USA
}

\begin{abstract}
A model device to transport thermal ions in the cyclotron gas stopper, a next-generation beam thermalization device under construction at the National Superconducting Cyclotron Laboratory, is presented. Radioactive ions produced by projectile fragmentation will come to rest at distances as large as $45 \mathrm{~cm}$ from the extraction orifice of the cyclotron gas stopper. The thermalized ions will be transported to the exit by RF carpets employing the recently developed "ion surfing" method. A quarter-circle prototype RF carpet was tested with potassium ions, and ion transport velocities as high as $60 \mathrm{~m} / \mathrm{s}$ were observed over distances greater than $10 \mathrm{~cm}$ at a helium buffer gas pressure of 80 mbar. The transport of $\mathrm{K}$ ions from an RF carpet to an electrode below was also demonstrated. The results of this study formed the basis of the design of the RF carpets for use in the cyclotron gas stopper.
\end{abstract}

Keywords: RF carpet, Ion transport, Beam thermalization of projectile fragments, Radioactive ion beam manipulation, Traveling wave transport

\section{Introduction}

The development of the first generation of beam thermalization devices at the NSCL [1] have allowed, for the first time, high precision Penning trap mass measurements [2] at a fragmentation facility. Following this success, additional experimental programs that require high quality, low energy rare isotope beams, such as laser spectroscopy [3] and re-accelerated beam experiments, are being developed. This new range of experimental programs, combined with the future operation of the FRIB facility, have driven the development of more universal and efficient beam thermalization devices. The cyclotron gas stopper (CGS) $[4,5,6,7]$ represents such a next generation device. Its design will allow the delivery of shorter-lived and lighter mass nuclei compared to the present generation of linear gas cells. These new abilities are in part due to the application of a new ion transport method called "ion surfing" $[8,9,10]$. Results of the research and development of a full size quartercircle RF carpet integral to the design of the final RF carpets for the CGS will be presented.

\section{The Cyclotron Gas Stopper}

Currently, linear gas cells are the only devices available to thermalize beams of rare ions produced by projectile fragmentation $[1,11,12,13,14,15]$. In this method, solid degraders first remove most of the kinetic energy of the incident ions, and ion-buffer gas collisions in the gas cell dissipate the remaining energy. Although the thickness of the degrader can be adjusted to optimize the fraction of ions that are stopped in the buffer gas, the large momentum spread of the fragments generally prevents the thermalization of the entire ion beam in a finite volume.
An increase of either the buffer gas pressure or cell length could improve the stopping efficiency of the gas cell. However, both of these changes also increase the transport time of the ions through the cell: higher pressure decreases the repelling effect of the carpet and also results in increased collisions with buffer gas atoms which hinder the ions' motion through the cell, and a longer length increases the distance the stopped ions must traverse. Furthermore, an increased transport time of rare isotopes would result in undesirable losses by radioactive decay of the most exotic nuclei. Hence, the typical gas cell length is limited to $0.5-2.0 \mathrm{~m}[1,11,12,13,14,15]$.

The CGS will accommodate large momentum spreads and range straggling while keeping the transport time short. This is accomplished by wrapping the ions' trajectory in an inward spiral using a strong magnetic field. The beam will enter the device tangentially at the edge of the cell $[4,5,6,7]$. Since the ions make multiple circuits in the buffer gas as they spiral to a stop, the mass thickness that the ions encounter will be significantly larger than that of a linear gas cell. Simulations have shown that the typical stopping radius from the central extraction orifice is $\sim 30 \mathrm{~cm}$ regardless of ion identity [16]. Simulated ${ }^{79} \mathrm{Br}$ ions complete about 5 revolutions ending at an average radius of $\sim 35 \mathrm{~cm}$, which corresponds to a mass thickness of 18.5 $\mathrm{mg} / \mathrm{cm}^{2}$. Lighter ions will require more cycles to stop. For example, simulations predict that $74 \mathrm{mg} / \mathrm{cm}^{2}$ (20 revolutions at 30 $\mathrm{cm}$ and $120 \mathrm{mbar}$ ) are necessary to slow ${ }^{24} \mathrm{O}$ ions [16].

Once the ions have been thermalized in the gas, they will be transported to the center of the CGS by ion surfing RF carpets. The ion surfing method was proposed by Bollen [8], and ion transport efficiency and velocity measurements were performed with a linear prototype $[9,17]$ and recently with a circular de- 
vice [10]. RF carpets were first developed at RIKEN $[11,18]$ to transport ions in a buffer gas. RF carpets are 2D arrays of parallel or concentric electrodes. An oscillating RF electric field is applied to the array with each successive electrode phaseshifted by 180 degrees. The fluctuating electric field from adjacent electrodes results in a net repelling force that prevents slow-moving, positively charged ions from colliding with the electrodes. A push field, produced by a potential difference between the carpet and a positively charged plate facing the carpet, is employed to direct the ions towards the carpet surface, and the ions are confined in the potential minimum created by the opposing push and repelling forces. The ions can be transported by applying a static potential gradient along the carpet. To transport the ions with the ion surfing method, a lower amplitude and frequency signal is superimposed on the RF signal. The signal is phase-shifted by a fixed amount with a full cycle applied to at least three consecutive electrodes to generate a traveling wave signal. The quarter-circle prototype in this work had a wavelength of four electrodes, and the traveling wave signal was phase-shifted by 90 degrees on adjacent electrodes.

Once the ions reach the edge of the ion surfing RF carpets, the ions will be pulled down to a smaller RF carpet biased at a lower potential. The small carpet acts as part of the barrier between the buffer gas region inside the CGS and the low pressure region of the ion conveyor [19], which delivers the ions to the low energy beamlines. The smaller carpet directs the ions to the small orifice through which the ions exit the CGS due to the gas flow created by the pressure difference.

\section{RF Carpet Design and Measurement Methods}

The characteristics of the prototype circular carpet was based on the linear prototype RF carpet tested in Ref. [9]. Its parameters are as follows: pitch, $\mathrm{a}=0.375 \mathrm{~mm}$; gap-to-pitch ratio, $\gamma=0.67$; size $45 \mathrm{~cm}$ by $42 \mathrm{~cm}$ (see Figure 1 ). The carpet traces were segregated in two sections, with an inner section of radius $=29 \mathrm{~cm}$ and an outer section beginning at radius $=29$ $\mathrm{cm}$ and extending to radius $=40 \mathrm{~cm}$ as shown in Figure 1 . The carpet was split into two sections so that the carpet could be driven with two circuits, each connected to approximately onehalf of the total capacitive load. The measured capacitance of each section was found to be large (2.6 nF inner segment, 2.26 $\mathrm{nF}$ outer segment), and only the inner section of the carpet was driven with the same RF circuit originally used in Ref. [9] at a RF frequency of $\sim 8.5 \mathrm{MHz}$. Thus, only the inner region was used for the present measurements. The RF amplitude varied with radius from $53 \mathrm{~V}$ at the inner edge of the carpet to $70 \mathrm{~V}$ at the outermost electrodes. The RF amplitude was $\sim 65 \mathrm{~V}$ at the middle of the inner section, and this value is listed in all figures. Among the four sets of electrodes (one for each wave phase), the RF amplitudes varied as much as $15 \%$. Additional details of the experimental set-up are similar to those given in Ref. [9].

Two types of measurements were performed when characterizing the carpet: transport efficiency and velocity. Both measurements were performed following the procedure described in Ref. [9]. The transport efficiency along the carpet was determined by taking the ratio of the current collected at the edge

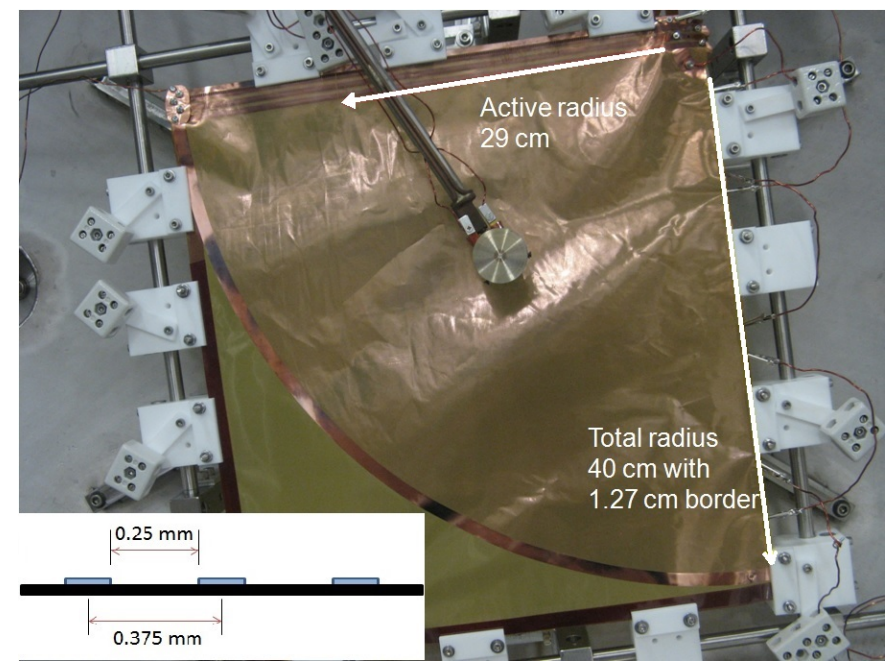

Figure 1: Photograph of the semi-circular RF carpet prototype mounted on a frame in the test chamber. The ions migrate from the ion source (circular object above the carpet) towards the upper right corner. The four bus bars that supply the RF and wave signals for each phase are visible along the upper edge. The radius of the inner, "active" area and the total radius of the carpet are indicated. A schematic of the cross section of three carpet traces is shown in the inset, with the carpet pitch $(\mathrm{a}=0.375 \mathrm{~mm})$ and gap $(\mathrm{g}=0.25 \mathrm{~mm})$ indicated.

of the carpet to the current from the ion source. The velocity measurements were taken by sending pulses of ions onto the carpet and measuring the travel time to the collection plate as a function of distance. The velocity measurements were taken at 5 positions in the region of $\mathrm{r}=10.6$ to $29 \mathrm{~cm}$.

\section{Experimental Results}

The two main parameters determining the transport velocities and efficiencies in the ion surfing method are the traveling wave amplitude and its velocity. As demonstrated in Ref. [9], the ion surfing transport efficiency is very sensitive to the traveling wave amplitude. This behavior was also investigated for the circular RF carpet in order to determine whether the linear [9] and circular carpets behave consistently.

The collection efficiency, $\eta$, is shown as a function of distance in Figure 2 for three wave amplitudes: 1.5, 2.5, and 3.5 $\mathrm{V}$. The half-distance, $\mathrm{x}_{0}$ is used to quantify the exponential decay in transmission efficiency in the expression $\eta=2^{-x / x_{0}}$, which was fitted to the experimental data. For small wave amplitudes $(<2.5 \mathrm{~V})$, the half-distances are quite large for the given parameters, much larger than the distance the ions will have to traverse to reach the CGS exit. At the relatively high amplitude of $3.5 \mathrm{~V}$, the half-distance decreased due to ion losses. When transported with a $3.5 \mathrm{~V}$ amplitude wave, the ions travel closer to the carpet surface and have a greater probability of being deflected into the carpet surface by a random collision with a buffer gas atom.

The maximum obtainable ion velocities as a function of push field and the corresponding wave velocity at 80 and 120 mbar are shown in Figure 3. In these measurements, the transport efficiency had to be at least $90 \%$ over $10 \mathrm{~cm}$, roughly correlating to a half-distance of $0.5 \mathrm{~m}$. Note that at push fields of $10 \mathrm{~V} / \mathrm{cm}$ 


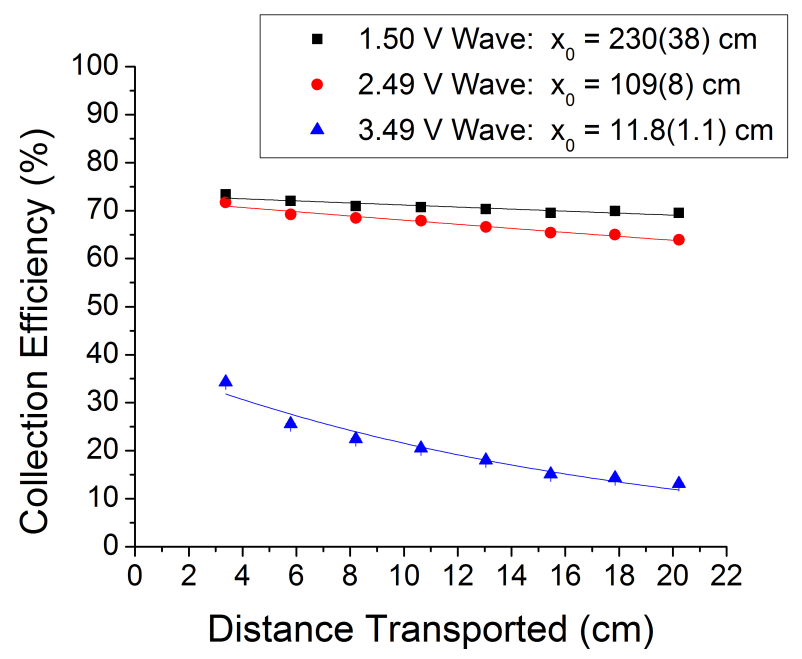

Figure 2: Collection efficiency as a function of the distance traveled by $\mathrm{K}^{+}$ions at three different wave amplitudes. The solid lines represent the best fit of the function $\eta=2^{-x / x_{0}}$ to the data, and the half distances, $\mathrm{x}_{0}$, are listed on the graph. Parameters: a $=0.375 \mathrm{~mm}, \gamma=0.67,80 \mathrm{mbar}, 20 \mathrm{~V} / \mathrm{cm}$ push field, 8.47 $\mathrm{MHz}$ RF frequency, $65 \mathrm{~V}$ RF amplitude, and $60 \mathrm{~m} / \mathrm{s}$ wave velocity.

or greater, the ions cannot efficiently travel in "locked mode," in which the ions travel at the wave velocity, but rather in "slipping" mode, $v_{\text {ion }}<v_{\text {wave }}$. The ions only reach locked mode with a $5 \mathrm{~V} / \mathrm{cm}$ push field at $80 \mathrm{mbar}$. In the CGS, the ions will be transported over a maximum of $0.5 \mathrm{~m}$ dictated by the overall design of the device. Recall that the simulated stopping radius for $\mathrm{A}=40$ ions in the CGS is about $25 \mathrm{~cm}$, so that the extraction times at the maximum speeds are predicted to be $\sim 4 \mathrm{~ms}$ at $80 \mathrm{mbar}$ and $\sim 20 \mathrm{~ms}$ at $120 \mathrm{mbar}$. The ion velocity is lower at 120 mbar due to the lower traveling wave velocity required to achieve efficent transport. These extraction times are excellent compared to the extraction times of previous and current beam thermalization devices $[6,20]$. Additionally, the experimental results show a trade-off between ion transport time and efficiency, which can be adjusted to optimize the delivery of the ion of choice.

\section{Ion Jumping Experiments}

A small $(\mathrm{r}=2.74 \mathrm{~cm}) \mathrm{RF}$ carpet with a rigid substrate will be placed a short distance below the center of the larger Kaptonbased RF carpet to collect the ions at the smallest radii. Geometric and mechanical considerations that drive the need for a small central carpet are as follows: the larger upper carpets must be cut at a radius larger than the size of the exit hole, the substrate of the carpet surrounding the exit hole must be strong enough to form the barrier between the gas chamber and the vacuum of the beam transport line, a second carpet allows verification of ion transport to the center of the cyclotron gas stopper, and the fields near the center of the device can be re-shaped by replacing only the central carpet.

Ion jumping from upper to lower carpet with drag field carpets was previously demonstrated [21]. Similar experiments were conducted with $\mathrm{Rb}$ ions for ion surfing carpets as part of

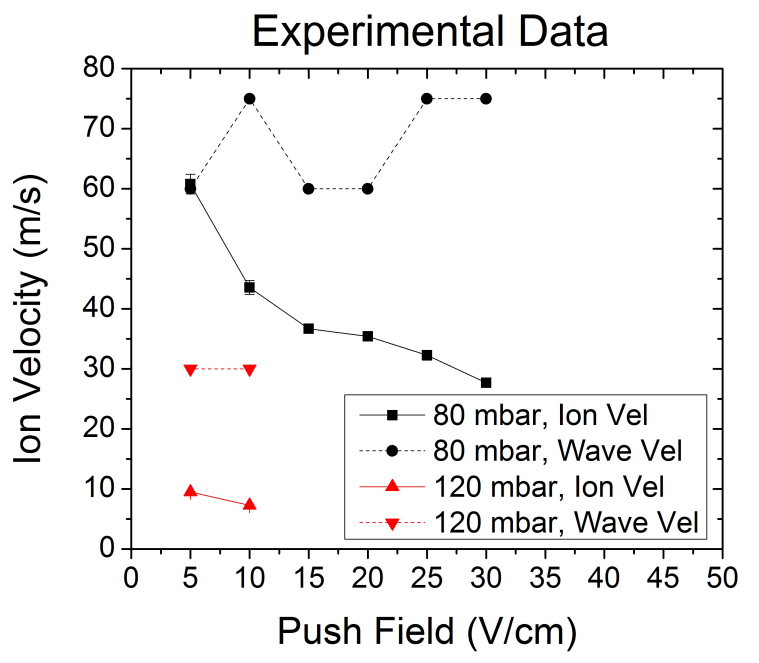

Figure 3: The maximum ion transport velocities (solid lines) with a half distance greater than $0.5 \mathrm{~m}(\sim 90 \%$ transport over $10 \mathrm{~cm})$ for $\mathrm{K}^{+}$ions are compared with the wave velocities (dashed lines). Parameters: $\mathrm{a}=0.375 \mathrm{~mm}, \gamma=0.67$, 8.47 MHz and 65 V RF amplitude.

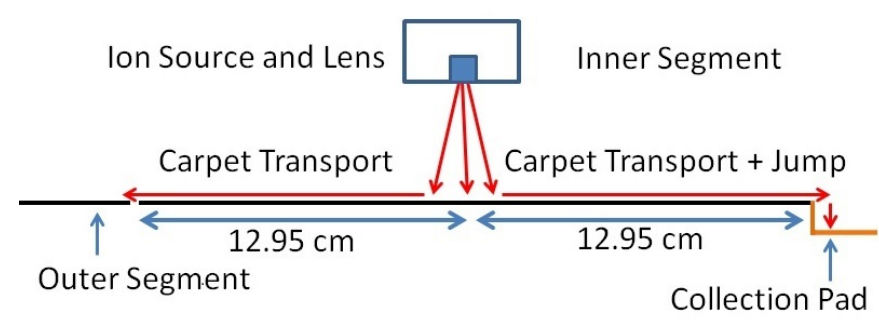

Figure 4: Cartoon of the cross section of the semi-circular prototype during ion jumping measurements. The carpet is indicated by black line and the collection pad by orange lines. The source was located over the midpoint of the inner segment. The collection pad was $1.2 \mathrm{~cm}$ below the large carpet.

this work. The quarter-circle carpet was modified for the jumping experiments, as shown in Figure 4. The central collection pad was removed and replaced with a collection pad placed 1.2 $\mathrm{cm}$ below the carpet surface. The new collection pad and outer segment of the carpet were connected to picoammeters, and a variable bias was applied to the collection pad. The potential difference between the voltage-biased collection pad and the upper carpet at ground created a pull field. The ion source was placed in the center of the active region of the carpet, equidistant $(12.95 \mathrm{~cm})$ from the inner edge of the carpet and the outer segment. The jumping efficiency was taken as the ratio of the measured transport efficiency to the collection pad below the carpet to that of the transport efficiency to the outer edge of the carpet, with the outer segment acting as a collection pad. The ion transport direction was easily switched by reversing the wave velocity, and each measurement was taken twice.

Various buffer gas pressures, as well as push and pull fields, were investigated in the jumping experiments. $75 \mathrm{~m} / \mathrm{s}$ was chosen as the wave velocity due to the excellent velocity and efficiency values observed for $\mathrm{Rb}$ [9], and a wave amplitude of $1.2 \mathrm{~V}$ was selected to optimize the transport efficiency so that 

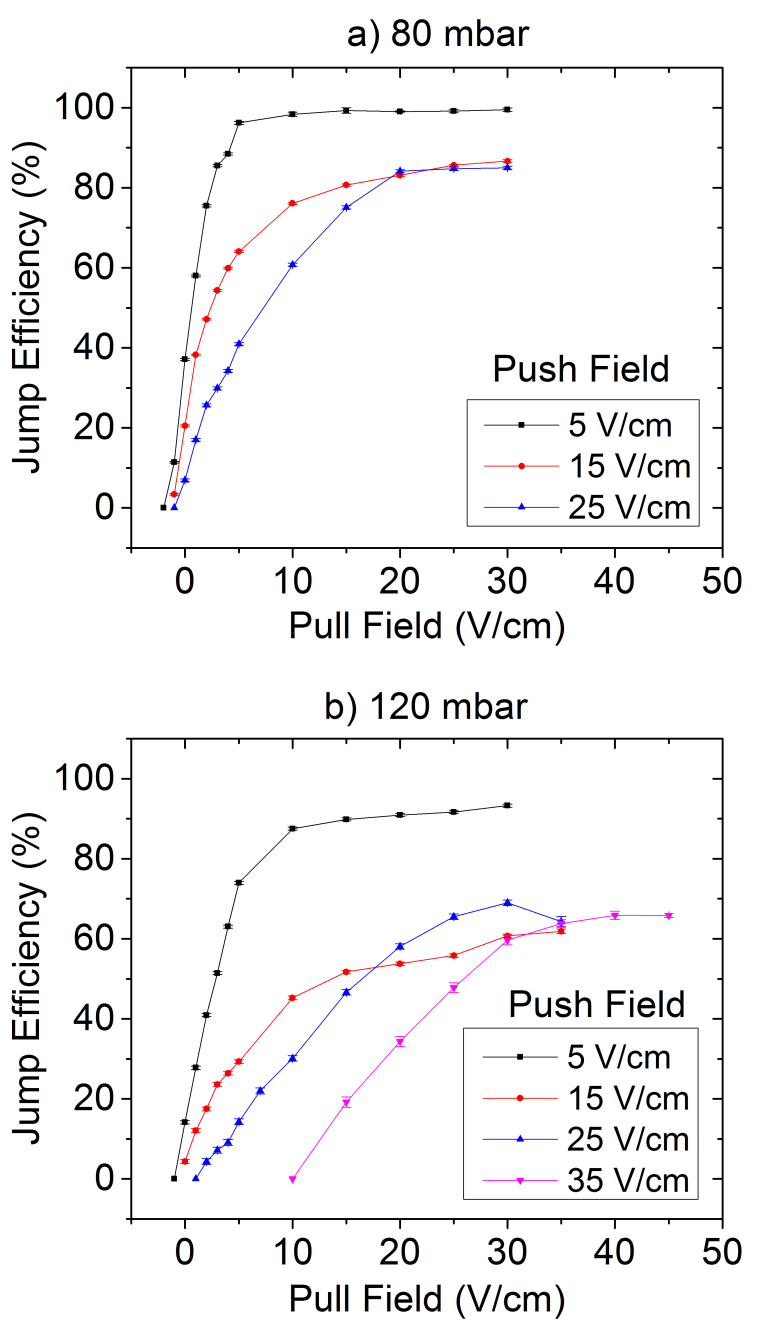

Figure 5: Jumping efficiency as a function of pull field for $\mathrm{Rb}^{+}$ions obtained at a) 80 mbar and b) 120 mbar at different push fields. Parameters: $a=0.375$ $\mathrm{mm}, \gamma=0.67,8.47 \mathrm{MHz} \mathrm{RF}$ frequency, $65 \mathrm{~V} \mathrm{RF}$ amplitude, $75 \mathrm{~m} / \mathrm{s}$ and $1.2 \mathrm{~V}$ amplitude traveling wave.

losses due to jumping would be readily observable.

The ion jumping results as a function of pull field at 80 mbar and 120 mbar are shown in Figure 5 for various push fields. At $80 \mathrm{mbar}$ and $5 \mathrm{~V} / \mathrm{cm}$ push field, nearly all of the ions were successfully transported to the collection pad with pull fields greater than $10 \mathrm{~V} / \mathrm{cm}$. At the higher push fields, the jumping efficiency at 80 mbar saturated at $\sim 80 \%$. As the push field increased a greater pull field was required to reach the maximum (saturated) efficiency. The ion losses most often occurred on the last carpet electrode in simulations. The lack of electrodes after the last one results in RF field asymmetry at the inner edge of the carpet and weaker repelling force.

At 120 mbar, the best results were again obtained at the lowest push field of $5 \mathrm{~V} / \mathrm{cm}$ and pull fields of $10 \mathrm{~V} / \mathrm{cm}$ or greater; however, the maximum efficieny was reduced to $90 \%$. The jump efficiency at 120 mbar saturated at $\sim 65 \%$ for the higher push fields, and again higher pull fields were required with increasing push field to reach the saturation efficiency. The results at both pressures show that a low push field in the center of the CGS is desirable to optimize jumping efficiency. Further tests will be conducted with a RF carpet replacing the collection pad below the upper carpet. The tests will ascertain that the ions will be repelled by the lower carpet's RF potential so that the ions will not terminate on its surface.

\section{Conclusion}

A quarter-circle prototype RF carpet was designed, fabricated, and tested. Valuable insight was gained concerning the design and mounting of the final RF carpets, especially in regards to the capacitance. $\mathrm{K}^{+}$ions were transported quickly and efficiently with predicted CGS extraction times reaching unprecedented fast times of $\sim 4 \mathrm{~ms}$ at 80 mbar buffer gas pressure and $\sim 20 \mathrm{~ms}$ at $120 \mathrm{mbar}$. $\mathrm{Rb}^{+}$ions were successfully transported from the quarter-circle prototype to a collection pad below the carpet, and optimal conditions for this necessary extraction step were investigated. The final large-area carpets, which are one-sixth of a circle with a radius of $42 \mathrm{~cm}$ and a pitch of $0.467 \mathrm{~mm}(\gamma=0.73)$, have been fabricated and are currently being prepared for use in the cyclotron gas stopper.

\section{Acknowledgements}

This work was supported by the National Science Foundation under grants PHY-09-58726 and PHY-11-02511.

\section{References}

[1] L. Weissman et al., Nucl. Phys. A 746 (2004) 655-658.

[2] G. Bollen, Acta. Phys. Pol. B 39 (2008) 433-444.

[3] K. Minamisono et al., Proceedings of the Institute for Nuclear Theory 16 (2009) 180.

[4] G. Bollen et al., Nucl. Instrum. Meth. A 550 (2005) 27-38.

[5] C. Guénaut et al., Hyperfine Interactions 173 (2006).

[6] G. Bollen et al., Nucl. Instrum. Meth. B 266 (2008) 4442-4448.

[7] S. Schwarz et al., Nucl. Instrum. Meth. B 317, Part B (2013) 463-467.

[8] G. Bollen, Int. J. Mass. Spectrom. 299 (2011) 131-138.

[9] M. Brodeur et al., Int. J. Mass. Spectrom. 336 (2013) 53-60.

[10] F. Arai et al., Int. J. Mass. Spectrom. 362 (2014) 56-58.

[11] M. Wada et al., Nucl. Instrum. Meth. B 204 (2003) 570-581.

[12] W. Trimble et al., Nucl. Phys. A 746 (2004) 415-418.

[13] M. Ranjan et al., EPL 96 (2011) 52001.

[14] W.R. Plaß et al., Nucl. Instrum. Meth. B 317, Part B (2013) 457-462.

[15] S. Purushothaman et al., Europhys. Lett. 104 (2013) 42001.

[16] N. Joshi et al., Proc. IPAC Conf. 2012, New Orleans, LA (2012) 2029.

[17] A. E. Gehring, Ph.D. Dissertation (2013).

[18] M. Wada, Nucl. Instrum. Meth. A 532 (2004) 40-47.

[19] M. Brodeur et al., Nucl. Instrum. Meth. A 317, Part B (2013) 468-472.

[20] M. Wada, Nucl. Instrum. Meth. B 317, Part B (2013) 450-456.

[21] G. K. Pang, Ph.D. Dissertation (2011). 